\title{
Routing in Telecommunication Networks Using Fuzzy Logic
}

\author{
Andriy A. Semenov ${ }^{1}$, Graduate Student Member, IEEE, Olena O. Semenova ${ }^{1}$, \\ Oleksandr M. Voznyak ${ }^{1}$, Oleksandr M. Vasilevskyi ${ }^{1}$, Maksym Yu. Yakovlev ${ }^{2}$ \\ ${ }^{1}$ Vinnytsia National Technical University, Vinnytsia, Ukraine \\ ${ }^{2}$ Hetman Petro Sahaidachny National Army Academy, Lviv, Ukraine
}

\begin{abstract}
In the paper it has been proposed to use a fuzzycontroller in telecommunication networks for improving the routing process. An architecture of the fuzzy-controller was developed. Linguistic variables, terms and membership functions for input and output values have been defined. Rules base has been developed. The operation of the fuzzy-controller has been simulated
\end{abstract}

Index Terms - fuzzy, telecommunication, routing.

\section{INTRODUCTION}

$\mathrm{N}$ OWADAYS telecommunication networks provide with more and more services for the multimedia and mobile data transmission. So, in order to provide efficient data forwarding, modern routing protocols are needed, because the networks become more complex and consist of a great deal of devices. Also, they must work in different conditions. Moreover, topology of a telecommunication network can change rapidly and it needs allowing for when developing the efficient routing algorithms.

Routing algorithms refer to a process of finding appropriate path among existing ones. Often simple metrics like the shortest path, signal strength, or node battery's residual are used to construct the route for data transmission. In modern telecommunication networks such as MANET or WSN the battery powered devices, namely laptops, smartphones and others are widely used as mobile nodes. Because of the limited energy resources of these devices existing routing strategies should be adapted in order to preserve the node energy. However, the existing routing algorithms are mostly based on the residual energy of nodes for selecting the next hop.

The route selection scheme efficiency can be improved by applying modern intelligent tools to choose the most optimal route [1-5]. Soft computing techniques, such as fuzzy logic, neural networks, genetic algorithms, have replaced conventional techniques in many engineering applications, especially in control systems and have been proved to be very effective.

Fuzzy logic system is able for reasoning under uncertainty implementing human experiences. Thus, fuzzy logic can be used when dealing with uncertain information while a network shows dynamic nature.

Thus, the aim of this work is improving the efficiency routing in telecommunication networks accounting for the fuzzy logic application.

\section{ARCHITECTURE OF THE FUZZY-CONTROLLER}

Generally, a fuzzy controller consists of four main blocks: a fuzzifier, defuzzifier, inference engine, and fuzzy rule base. The fuzzifier transforms each input variable to the membership functions values. The inference engine calculates the fuzzy output depending on the rule base. The defuzzifier converts the fuzzy output to crisp value using mathematical formulas.

To construct a fuzzy system, the input and output linguistic variables should be defined as membership functions. The membership function is a graphical interpretation of the input and output linguistic variables. Then fuzzy rules (IFTHEN) connecting the input memberships with the output membership should be suggested.

We propose to use in telecommunication networks a fuzzy-controller having three input and one output linguistic variables (Fig.1). Input linguistic variables of the fuzzycontroller are the energy (E), a number of hops $(\mathrm{H})$, and a distance (D), its output variable is a chance of receiving (C). Energy is the residual energy spent by each node for transmitting and receiving packets. The number of hops to the node represents the number of times that data must be forwarded to reach the destination node. The distance is threedimensional distance to the next node. Other parameters such as channel properties or network congestion also can be used after adapting the fuzzy sets and rules proposed. The defuzzified value of the output fuzzy variable, the chance of receiving, determines the chance for forwarding neighbor node to be selected for the next hop.

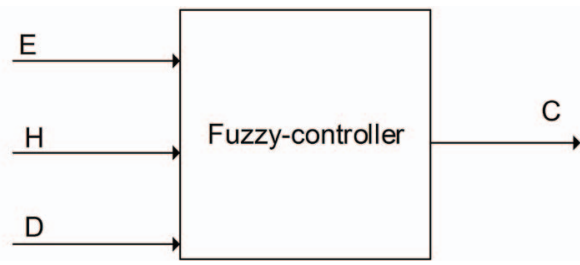

Fig.1. The architecture of the fuzzy-controller

For defining energy E terms "low", "medium", and "high" are used. Thus, the term set of $\mathrm{E}$ is:

$$
T(E)=\{\operatorname{Low}(L), \text { Medium }(M), \operatorname{High}(H)\} .
$$

Membership functions for T(E) are presented on Fig. 2. 
$\mathrm{T}(\mathrm{E})$

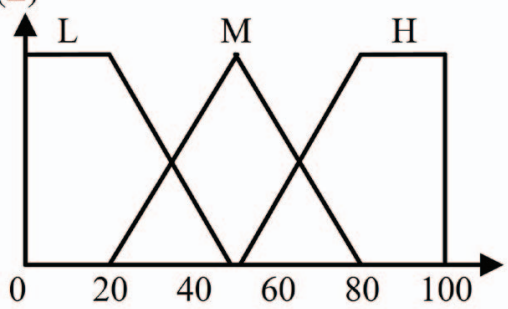

Fig.2. Membership functions for the E linguistic variable

For defining the number of hops H terms "few", "several" and "many" are used. Thus, the term set of $\mathrm{H}$ is:

$$
T(H)=\{\text { Few }(F), \text { Several }(S), \text { Many }(M)\}
$$

Membership functions for $\mathrm{T}(\mathrm{H})$ are presented on Fig. 3. For defining the distance D terms "near", "middle", and "far" are used. Thus, the term set of D is:

$$
T(D)=\{\operatorname{Near}(N), \text { Middle }(M), \text { Far }(F)\} .
$$

Membership functions for T(D) are presented on Fig. 4. For defining the chance of receiving $\mathrm{C}$ terms "very low", "low", "medium", "high" and "very high" are used. Thus, the term set of $\mathrm{C}$ is:

$$
T(C)=\{\operatorname{Very} \text { low }(V L), \text { Low }(V),
$$

Medium $(M)$, High $(H)$,Very high $(V H)\}$.

Membership functions for $\mathrm{T}(\mathrm{C})$ are presented on Fig. 5.

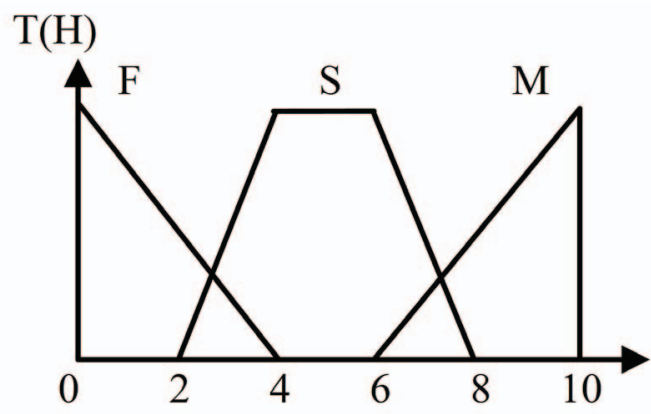

Fig.3. Membership functions for the $\mathrm{H}$ linguistic variable

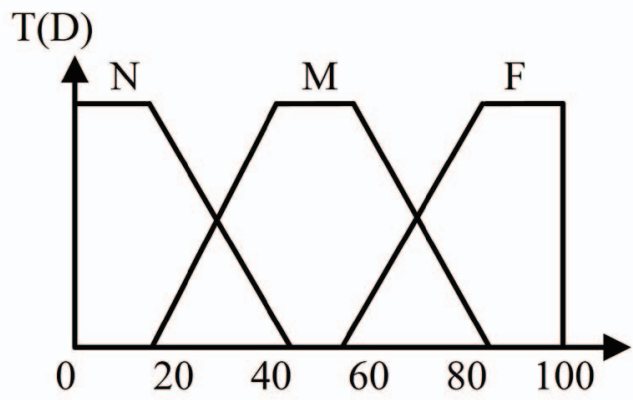

Fig.4. Membership functions for the D linguistic variable

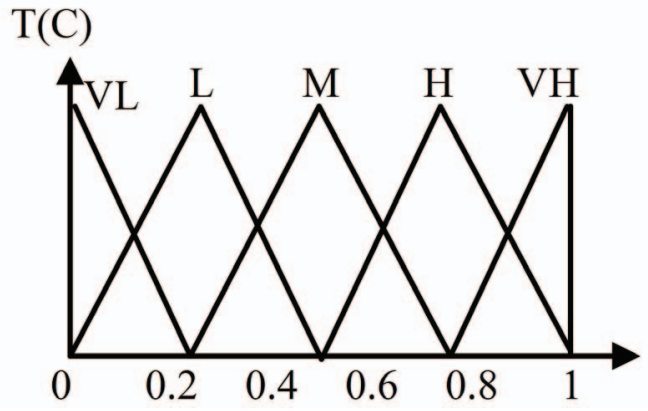

Fig.5. Membership functions for the $\mathrm{C}$ linguistic variable

The proposed fuzzy controller works according to a rule base consisting of twenty-seven rules:

1. If $E=L$ and $H=F$ and $D=N$ than $C=L$;

2. If $E=L$ and $H=F$ and $D=M$ than $C=L$;

3. If $E=L$ and $H=F$ and $D=F$ than $C=L$;

4. If $E=L$ and $H=S$ and $D=N$ than $C=L$;

5. If $E=L$ and $H=S$ and $D=M$ than $C=V L$;

6. If $E=L$ and $H=S$ and $D=F$ than $C=V L$;

7. If $E=L$ and $H=M$ and $D=N$ than $C=V L$;

8. If $E=L$ and $H=M$ and $D=M$ than $C=V L$;

9. If $E=L$ and $H=M$ and $D=F$ than $C=V L$;

10. If $E=M$ and $H=F$ and $D=N$ than $C=H$;

11. If $E=M$ and $H=F$ and $D=M$ than $C=H$;

12. If $E=M$ and $H=F$ and $D=F$ than $C=M$;

13. If $E=M$ and $H=S$ and $D=N$ than $C=M$;

14. If $E=M$ and $H=S$ and $D=M$ than $C=M$;

15. If $E=M$ and $H=S$ and $D=F$ than $C=M$;

16. If $E=M$ and $H=M$ and $D=N$ than $C=M$;

17. If $E=M$ and $H=M$ and $D=M$ than $C=L$;

18. If $E=M$ and $H=M$ and $D=F$ than $C=L$;

19. If $E=H$ and $H=F$ and $D=N$ than $C=V H$;

20. If $E=H$ and $H=F$ and $D=M$ than $C=V H$;

21. If $E=H$ and $H=F$ and $D=F$ than $C=V H$;

22. If $E=H$ and $H=S$ and $D=N$ than $C=V H$;

23. If $E=H$ and $H=S$ and $D=M$ than $C=V H$;

24. If $E=H$ and $H=S$ and $D=F$ than $C=H$;

25. If $E=H$ and $H=M$ and $D=N$ than $C=H$;

26. If $E=H$ and $H=M$ and $D=M$ than $C=H$;

27. If $E=H$ and $H=M$ and $D=F$ than $C=H$. 


\section{OPERATION OF THE FUZZY-CONTROLLER}

The proposed fuzzy-controller operates as follows.

In the fuzzyfier each input variable gets a corresponding fuzzy value:

$$
\begin{gathered}
E \Rightarrow E_{L}, E_{M}, E_{H} ; \\
H \Rightarrow H_{F}, H_{S}, H_{M} ; \\
D \Rightarrow D_{N}, D_{M}, D_{F}
\end{gathered}
$$

Then in the inference engine the minimum are performed:

$$
\begin{gathered}
w_{1}=\min \left[E_{L}, H_{F}, D_{N}\right], \\
w_{2}=\min \left[E_{L}, H_{F}, D_{M}\right], \\
\ldots \\
w_{27}=\min \left[E_{H}, H_{M}, D_{F}\right] .
\end{gathered}
$$

In the defuzzyfier the crisp value is obtained:

$$
C=\frac{\sum_{i=1}^{27} w_{1} \cdot C_{1}}{\sum_{i=1}^{27} w_{1}} .
$$

\section{SIMULATION}

In order to confirm the operability of the fuzzy-controller we can use the Matlab 6.5 program. The simulated fuzzycontroller is shown on Fig.6.

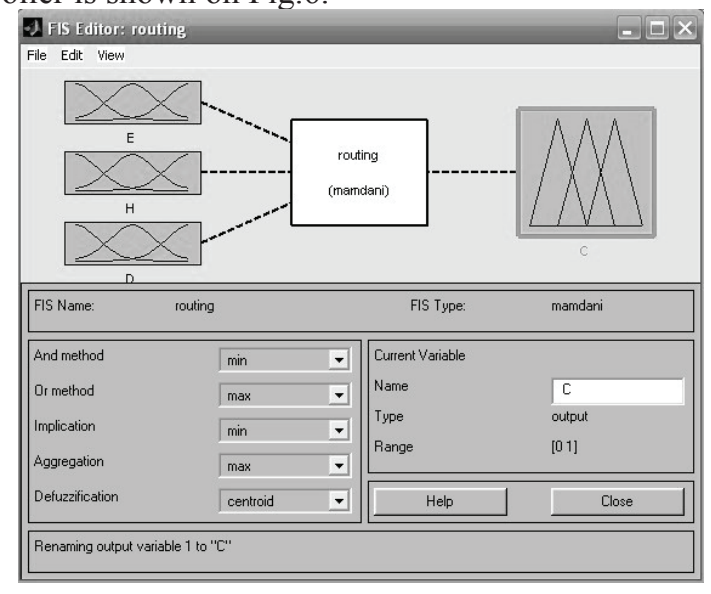

Fig.6. The fuzzy-controller in Matlab 6.5

Specifying the energy linguistic variable is shown on Fig.7.

Specifying the number of hops linguistic variable is shown on Fig. 8.

Specifying the distance linguistic variable is shown on Fig.9.

Specifying of the chance of receiving output linguistic variable is shown on Fig. 10.

Specifying of the rule base is shown on Fig.11.

The rule surface is shown on Fig. 12.

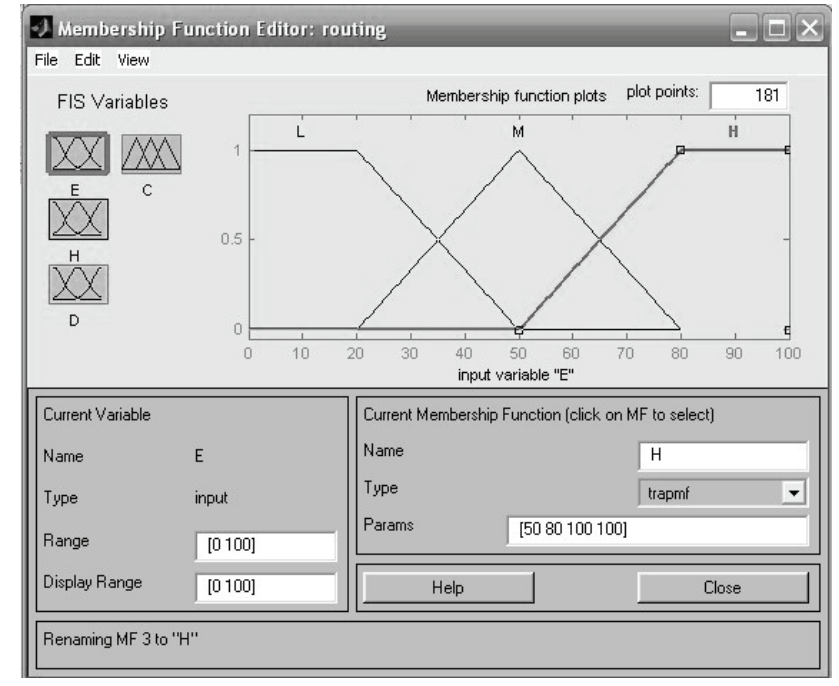

Fig. 7. Specifying of the E input variable

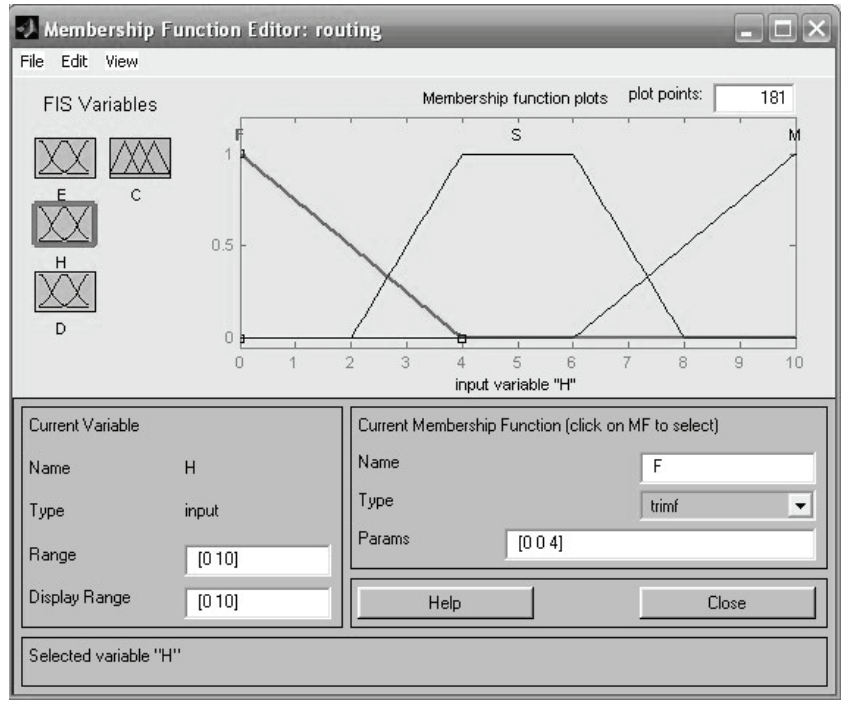

Fig. 8. Specifying of the $\mathrm{H}$ input variable

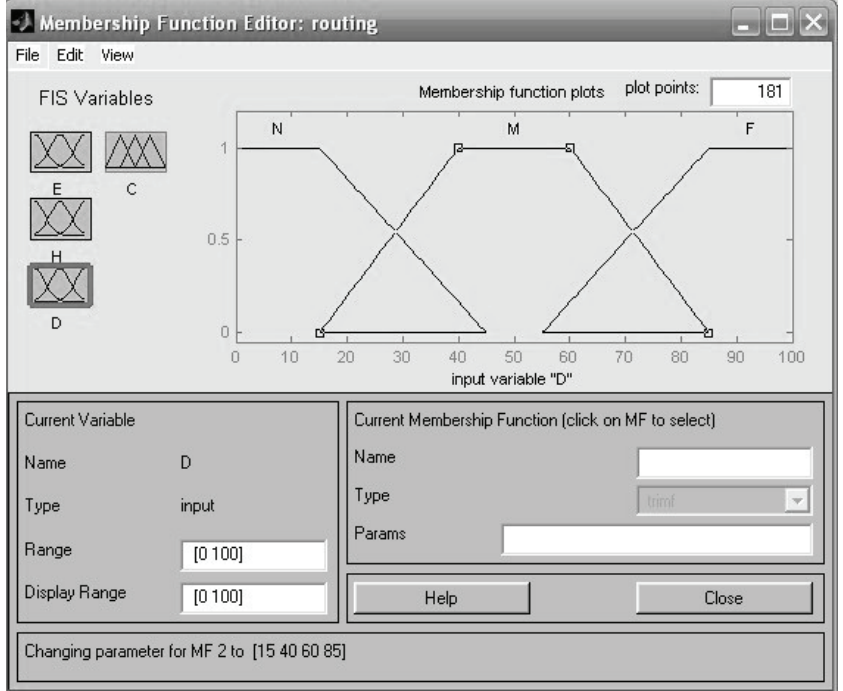

Fig. 9. Specifying of the D input variable 


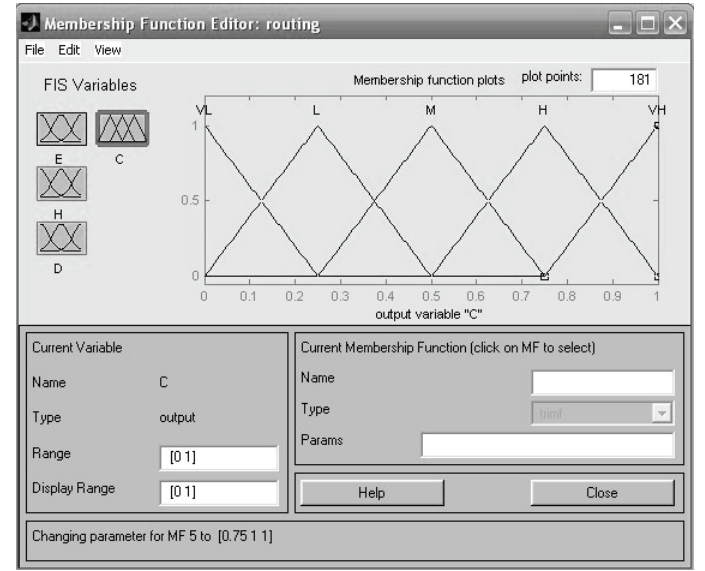

Fig.10. Specifying of the $\mathrm{C}$ output variable

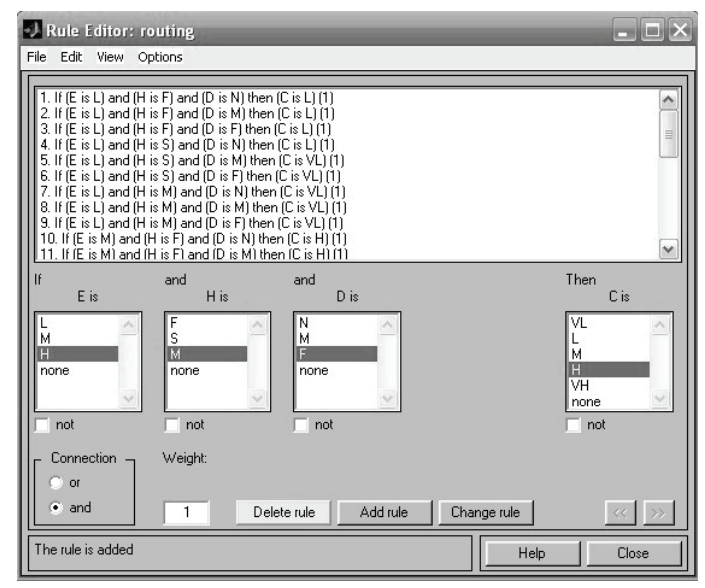

Fig. 11. The rule base

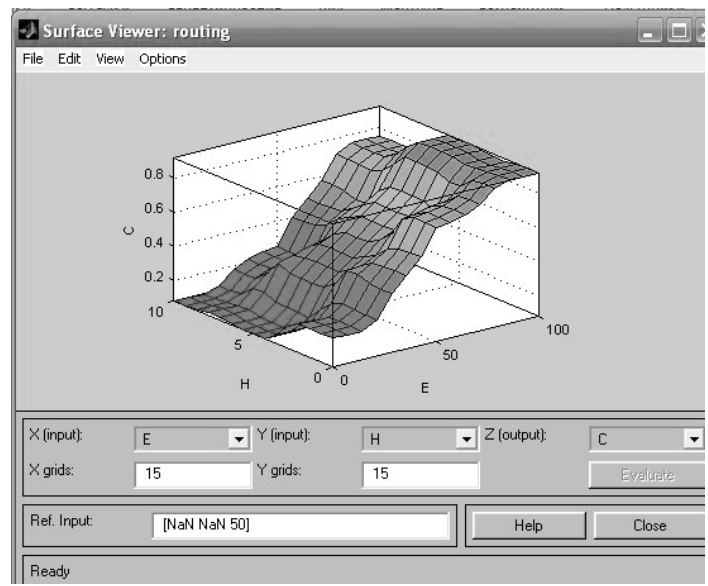

Fig.12. The rule surface

Let the energy $\mathrm{E}=20$, the number of hops $\mathrm{H}=7$, and the distance $\mathrm{D}=50$. According to Fig. 13 we get the chance of receiving $\mathrm{C}=0.095$.

Let the energy $\mathrm{E}=30$, the number of hops $\mathrm{H}=1$, and the distance $\mathrm{D}=20$. According to Fig. 14 we get the chance of receiving $\mathrm{C}=0.44$.

Let the energy $\mathrm{E}=80$, the number of hops $\mathrm{H}=10$, and the distance $\mathrm{D}=40$. According to Fig. 15 we get the chance of receiving $\mathrm{C}=0.75$.

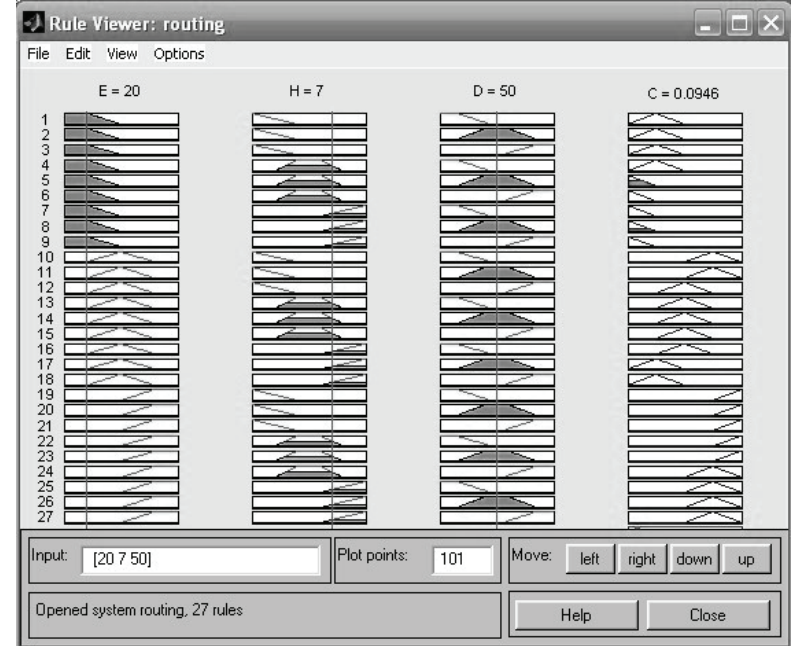

Fig.13. The simulation result

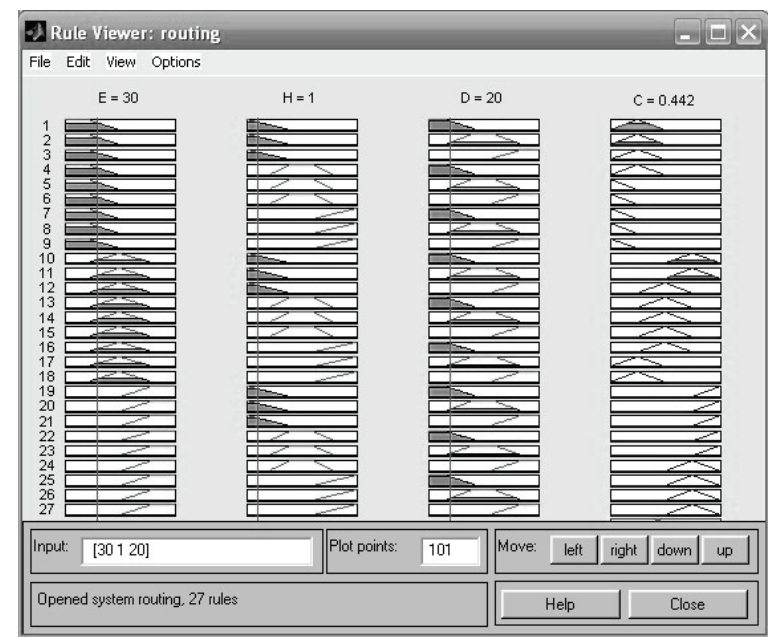

Fig.14. The simulation result

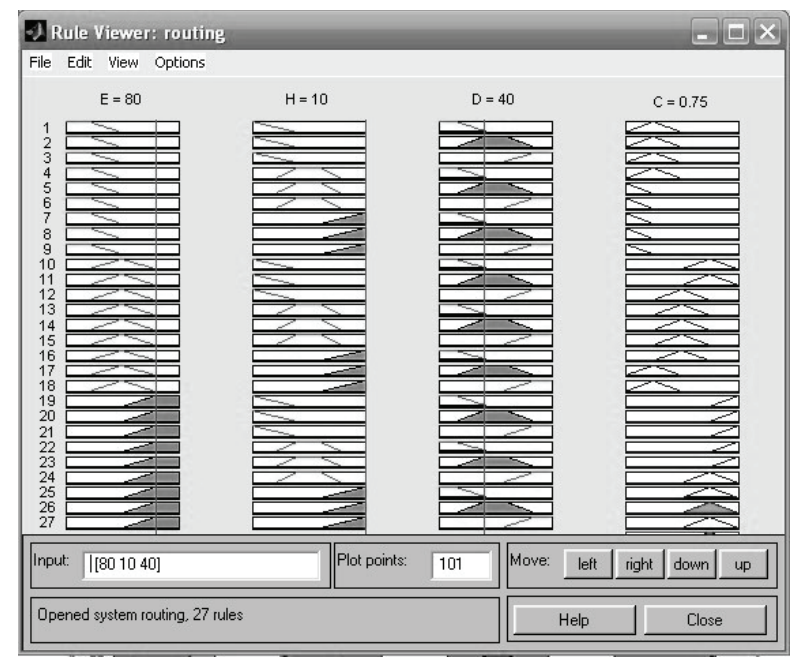

Fig.15. The simulation result

So, according to the simulation results, the proposed routing fuzzy-controller can be applied in telecommunication systems. 


\section{CONCLUSION}

In this work, the fuzzy-controller is proposed as an approach to compute a node's change of data receiving. It provides an efficient routing scheme by selecting the most trustworthy nodes for constructing a stable route. Also, it preserves resources of the network and improves its performance.

\section{REFERENCES}

[1] Atayero A. A Applications of Soft Computing in Mobile and Wireles Communications / Atayero A. A., Luka M. K. // International Journal of Computer Applications. - Vol. 45. - №. 22. - 2012. - Pp. 48-54

[2] Kumar V. B. P. Neural Networks Based Efficient Multiple Multicast Routing for Mobile Networks / Kumar V. B. P., Kumar D. S. M. // International Journal of Information and Electronics Engineering. vol. 4. - №. 2. -2014 - pp. 145-152.

[3] Kojic, N. New Algorithm for Packet Routing in Mobile Ad-hoc Networks / N. S. Kojic, M. B. Z. Ivancic, I. S. Reljin, B. D. Reljin // Journal of automatic control. University of Belgrade. - Vol.20. 2010. - Pp. 9-16.

[4] Alandjani G. / Fuzzy routing in ad hoc networks / Alandjani G. Johnson E. E. // Proceedings of the 2003 IEEE International Performance, Computing, and Communications Conference. - 2003 - Pp. 525 - 530

[5] Anecia C. M. FACM: Fuzzy Based Resource Admission Control For Multipath Routing in MANET / Anecia C. M., Pushpalatha M. S. // International Journal of Advanced Research in Computer Science \& Technology (IJARCST 2014) - Vol. 2. - 2014 . - pp. 114-119.

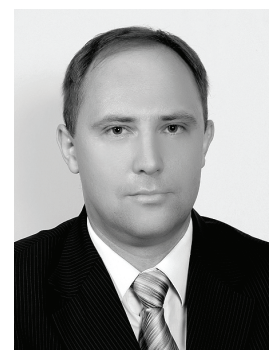

Andriy O. Semenov is Professor in the Department of Radio Engineering, Faculty for Radio Engineering, Telecommunication and Electronic Instrument Engineering, Vinnytsia National Technical University, Vinnytsia, Ukraine. He received Master degree in Radio Engineering, and $\mathrm{PhD}$ degree in Engineering from Vinnytsia National Technical University, Vinnytsia, Ukraine. He is working toward the D.Sc degree. IEEE member since 2015. He has 177 academic and research publications. His areas of research interest include signal oscillating and non-linear dynamics.

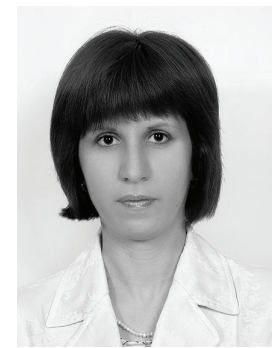

Olena O. Semenova is Associate Professor in the Department of Telecommunication Systems and Television, Faculty for Radio Engineering, Telecommunication and Electronic Instrument Engineering, Vinnytsia National Technical University, Vinnytsia, Ukraine. She received Bachelor Degree in Radio Engineering, Master degree in Radio Engineering, and $\mathrm{PhD}$ degree in Engineering from Vinnytsia National Technical University, Vinnytsia, Ukraine. She has 94 academic and research publications. Her areas of research interest include telecommunication networks and soft computing.

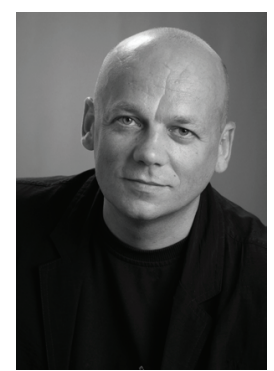

Oleksandr M. Voznyak is Associate Professor in the Department of metrology and industrial automatics, Faculty for Computer Systems and Automation, Vinnytsia National Technical University, Vinnytsia, Ukraine. He received and $\mathrm{PhD}$ degree in Engineering from Vinnytsia National Technical University, Vinnytsia, Ukraine. He has over 90 academic and research publications.

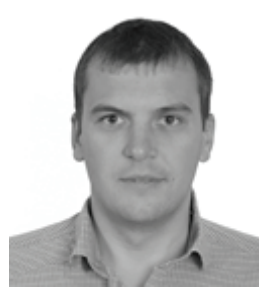

Oleksandr M. Vasilevskyi is Professor in the Department of Metrology and industrial automatics, Faculty for Computer Systems and Automation, Vinnytsia National Technical University, Vinnytsia, Ukraine. He received $\mathrm{PhD}$ and DSc degrees in Engineering from Vinnytsia National Technical University, Vinnytsia, Ukraine. He has over 150 academic and research publications.

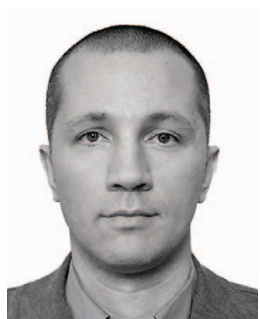

Maksym Yu. Yakovlev is DSc in Engineering, Senior Research Officer Deputy Head of the Scientific Center of the Land Forces, Hetman Petro Sahaidachny National Army Academy, Lviv, Ukraine. 